nation), or about every 700,000 base pairs. This density is much better than that specified in the goal for genetic mapping set for the US human genome programme, which was achieved more than a year ago. These markers have also been incorporated into the human physical map ${ }^{4-9}$.

Lander, Dietrich and colleagues' mouse genetic map marks the conclusion of a five-year project. This map also consists of microsatellite markers; but because mice can be bred at will, it is based on a cross between two strains of mice rather than on natural families. A total of 6,336 markers that are different in these strains were scored and assembled into a map of 1,001 'bins' containing an average of 6.3 markers each. Having completed this map, the authors then interdigitated it with a second mouse map based on 797 RFLPs and rich in known genes. The final integrated map has 7,377 loci, on average one every $0.2 \mathrm{cM}$ or every 400,000 base pairs.

Dense genetic maps make possible the identification of genes for single-gene disorders and the dissection of polygenic traits, and these two maps have already changed the face of human and mouse biology. Both groups made their markers available well before publication. So although these papers mark the completion of the maps, their impact has already permeated the genetics community.

Whether in mouse or man, mapping disease phenotypes by linkage analysis used to be a daunting task, one outside of the reach of many relatively sophisticated molecular biology laboratories. Marker sets were incomplete, the scoring required tedious and finicky Southern blotting methods, and years of work were often required to identify a linked marker. The new maps have changed all of that mapping a single-gene disorder (typically requiring analysis of tens of thousands of genotypes) can now be accomplished in a modest-sized lab in a few months.

For polygenic conditions, the challenges are much greater, as the simultaneous tracking of several susceptibility loci, each making only a relatively modest contribution to risk of disease, requires much larger numbers of affected individuals. Such projects often need hundreds of thousands of genotypes, and can only be attempted with the kind of dense and readily scorable marker maps described here. This incipient harvest of information about genetic contributions to complex traits, much anticipated as one of the most powerful components of the new molecular medicine, is now beginning to appear. Many of the early successes, from mapping loci involved in hypertension to mapping those for certain behavioural traits, have been achieved in animal models because of the additional analytical power made possible by selective breeding schemes. Thanks to the availability of these maps, however, promising chromosomal regions associated with polygenic human disorders such as juvenile-onset diabetes, schizophrenia and learning disability are now being identified. A rat genome project has recently been spun off, the reason being that a large number of quantitative trait models are available in that species, and a similarly dense map of microsatellite markers for the rat is expected by 1998 .

What next for the Human Genome Project? The maps produced by Weissenbach, Lander and their colleagues have been compiled with care and devotion, and their value is unquestionable. Nonetheless, improvements in mapping technology may still be needed in the future. In particular, the need to run electrophoretic gels to separate microsatellite marker products by size presents obstacles to total automation of that process. Eventual replacement of these markers with an even denser map of single-nucleotide polymorphisms, which could be scored with DNA chip technology, may have significant advantages for truly highthroughput applications.

But with the genetic mapping goals essentially complete for now, we look forward to completion of the second phase, a physical map of the human genome, which is expected in about a year (see figure). This map will consist of 30,000 STS (sequence-tagged-site) markers distributed at intervals of approximately 100,000 bases along the entire genome. Physical maps of several chromosomes have been completed or are nearing completion, and late last year a preliminary physical map of the whole genome ${ }^{4}$ and a map with 94 per cent coverage from 15,000 markers $^{9}$ were published.

The international genome community is now turning its attention to the ultimate challenge, sequencing the 3 billion base pairs of the human genome. This year, a number of groups will be scaling up sequencing of human DNA to a rate of many megabases a year. There is growing confidence that this most difficult of the tasks, once considered impossible by many, will also be successfully accomplished - perhaps even a bit ahead of the original target date of 2005 .

Elke Jordan and Francis S. Collins are at the National Center for Human Genome Research, National Institutes of Health, Bethesda, Maryland 20892, USA.

\footnotetext{
1. Dietrich, W. F. et al. Nature 380, 149-152 (1996)

. Dib, C. et al. Nature 380, 152-154 (1996).

3. Murray, J. C. et al. Science 265, 2049-2054 (1994).

4. Chumakov, I. M. et al. Nature 377 Suppl. 175-297 (1995).

Gemmill, R. M. et al. Nature 377 Suppl. 299-319 (1995).

Krauter, K. et al. Nature 377 Suppl. 321-333 (1995).

7. Doggett, N. A. et al. Nature $\mathbf{3 7 7}$ Suppl. 335-365

(1995).

(1995).

8. Collins, J. E. et al. Nature 377 Suppl. 367-379 (1995).

9. Hudson, T. J. et al. Science 270, 1945-1954 (1995).
}

\section{Spin and lift}

A SPINNING ball swerves in the air - a fact known and exploited in many sports. This is the Magnus effect, by which a spinning object experiences lift at right-angles to the airflow past it. Curiously enough, even fixed-wing aircraft depend on the Magnus effect for their lift. It is generated by a flow of air around each wing, back over the top surface and forward again under the lower one. Of course, this circulation is merely a small component of the much larger slipstream flowing back past the craft. It takes the form of a slightly faster backflow over the wing, and a slightly slower one under it. Daedalus is now devising an improved wing with enormous Magnus circulation.

He recalls the Flettner sailing ship of the 1920s. Instead of sails, it had big upright spinning cylinders on its deck, which generated sideways Magnus thrust from the wind blowing past them. His first idea was an equivalent Flettner aircraft, with two long horizontal spinning cylinders sticking out from the fuselage instead of wings. Sadly, it would have far too much drag. But Daedalus soon saw a way out. Imagine, he says, a conventional aircraft wing, and imagine a continuous sheet wrapped round it like a wide conveyor belt. Spin this belt rapidly round the wing, back over the top and forward again underneath, and you have an ideal Magnus-effect wing.

Not only would this cunning design have far more lift than a standard wing; its lift could be varied in flight by changing the speed of the belt. A standard wing is crowded with elaborate slots, flaps and droops, all designed to raise its lift at low speeds, so as to simplify take-off and landing. Daedalus's light, elegant 'Magnaplane' needs no such complexity. Each wing is simply a frame carrying rollers defining a fairly conventional aerofoil cross-section, and a wide belt which runs round the rollers at a speed controlled by the pilot. Daedalus calculates that at their fastest, the racing belts could generate ten times the lift of a normal wing, or (just as useful) the same lift at a tenth of the speed.

Thus the Magnaplane combines the virtues of a biplane, a helicopter and a blimp. It can land and take off from tiny fields, saunter lazily around the sky on search or surveillance missions, turn on a dime and soar like a lift. Speed up the belt on one wing, and it will bank neatly for a turn; a bit more speed and it will flip into a spectacular roll. Put both belts into reverse, and it will drop like a stone in a awesome emergency dive. Even if the belts fail, it will merely revert to a conventional aeroplane, and can fly safely home. David Jones 